Revue musicale OICRM

revue musicale oicrm

\title{
Debus-si e(s)t Pierrot. Rire pour ne pas pleurer
}

\section{Benjamin Lassauzet}

Volume 2, numéro 2, 2015

Une relève

URI : https://id.erudit.org/iderudit/1060135ar

DOI : https://doi.org/10.7202/1060135ar

Aller au sommaire du numéro

Éditeur(s)

OICRM

ISSN

2368-7061 (numérique)

Découvrir la revue

Citer cet article

Lassauzet, B. (2015). Debus-si e(s)t Pierrot. Rire pour ne pas pleurer. Revue musicale OICRM, 2(2), 154-177. https://doi.org/10.7202/1060135ar

\section{Résumé de l'article}

L'une des premières œuvres humoristiques de Debussy est Pierrot (1882), une mélodie répétant sans cesse l'air populaire associé au personnage de la commedia dell'arte : « Au clair de la lune ». Comme de nombreux artistes de son temps, Debussy semble céder à la tentation de se projeter dans le personnage - ce à quoi invite le poème de Banville qu'il met en musique, soulignant cette ambigüité identitaire. Pierrot est aussi l'éternel perdant en amour, Colombine lui préférant toujours Arlequin. Or, Debussy dédie sa mélodie à Marie-Blanche Vasnier, sa première muse, une femme mariée dont il est tombé amoureux, lui offrant une nouvelle occasion de se trouver des points communs avec Pierrot. La musique confirme l'hypothèse de l'autoportrait, puisque Debussy donne un rôle de premier ordre à la note si, qu'il a choisie pour le représenter, correspondant à la dernière syllabe de son nom. Ainsi, derrière l'apparente légèreté, se cache la confession de la souffrance amoureuse de l'artiste. 


\title{
Debus-si e(s)t Pierrot. Rire pour ne pas pleurer Benjamin Lassauzet
}

\begin{abstract}
Résumé
L'une des premières œuvres humoristiques de Debussy est Pierrot (1882), une mélodie répétant sans cesse l'air populaire associé au personnage de la commedia dell'arte: "Au clair de la lune ». Comme de nombreux artistes de son temps, Debussy semble céder à la tentation de se projeter dans le personnage - ce à quoi invite le poème de Banville qu'il met en musique, soulignant cette ambigüité identitaire. Pierrot est aussi l'éternel perdant en amour, Colombine lui préférant toujours Arlequin. Or, Debussy dédie sa mélodie à Marie-Blanche Vasnier, sa première muse, une femme mariée dont il est tombé amoureux, lui offrant une nouvelle occasion de se trouver des points communs avec Pierrot. La musique confirme l'hypothèse de l'autoportrait, puisque Debussy donne un rôle de premier ordre à la note si, qu'il a choisie pour le représenter, correspondant à la dernière syllabe de son nom. Ainsi, derrière l'apparente légèreté, se cache la confession de la souffrance amoureuse de l'artiste.
\end{abstract}

Mots clés : autoportrait; commedia dell'arte ; Debussy ; humour musical ; Pierrot.

\begin{abstract}
One of the first comic works of Debussy's is Pierrot (1882), a melody consisting of the unceasing repetition of the popular song which is always associated to this commedia dell'arte character: "Au clair de la lune." Like many artists of his time, Debussy seems to have yielded to the temptation of clothing himself with Pierrot's costume-which is suggested by the poem written by Banville that he set to music, which underlines this identitary ambiguity. Pierrot is also the victim of a love triangle with Colombine and Harlequin - the female character always choosing the latter. Since Debussy dedicated this melody to Marie-Blanche Vasnier, his first muse, a maried woman he had fallen in love with, he found in the words of the poem some other elements in common with Pierrot. The music confirms this self-portrait hypothesis, since Debussy gave the leading role to the note B (in French, si), matching with the last syllable of his name (-ssy). Therefore, behind a seemingly light-hearted work, the artist hid his suffering for love.
\end{abstract}

Keywords: commedia dell'arte; Debussy; musical humor; Pierrot; self-portrait. 


\section{Debus-si e(s)t Pierrot : RIRE POUR Ne PAS Pleurer}

La publication, en supplément d'un numéro spécial de La Revue musicale de 1926 consacré à la jeunesse de Claude Debussy ${ }^{1}$, d'une mélodie jusque-là inédite du compositeur, a de quoi surprendre. D'une part parce que jamais auparavant il n'avait été question de ce Pierrot composé au début de l'année 1882. Et d'autre part parce qu'il donne du compositeur une image qui ne correspond guère à l'esthétique, tantôt impressionniste, tantôt symboliste, à laquelle on a l'habitude de rattacher Debussy ${ }^{2}$. En effet, il s'agit d'une œuvre comique, faisant entendre à l'envi la mélodie attitrée du personnage de la commedia dell'arte: "Au clair de la lune".

Bien que la paternité de cet air soit souvent attribuée - sans certitude - à JeanBaptiste Lully, il semblerait qu'il soit d'origine populaire : c'est en tout cas à cet environnement qu'il est communément associé. Mais le caractère atypique de cette œuvre ne repose pas sur la citation par un compositeur savant d'une mélodie populaire célèbre.

En effet, Debussy aura, à multiples reprises dans sa production, réitéré dans ce sens. Ainsi, entend-on « Dodo, l'enfant do » dans Jimbo's Lullaby (tiré du Children's Corner, dédié à sa fille Claude-Emma, dite Chouchou) ou dans l'introduction du ballet pour enfants La boîte à joujoux, œuvre qui cite également « Pan! Qu'est-ce qu'est là? » (tableau I), " Il était une bergère » (tableau III), « Fanfan la tulipe » (tableau IV)... La mélodie populaire la plus citée dans l'œuvre de Debussy étant sans doute « Nous n'irons plus au bois ", que l'on rencontre dans La belle au bois dormant (une mélodie sur un texte du chansonnier Vincent Hyspa), mais aussi Rondes de printemps (extrait des Images pour orchestre), et l'image (oubliée) Quelques aspects de Nous n'irons plus au bois

$1 \quad$ La Revue musicale $1926, \mathrm{n}^{\circ} 7$.

2 Appliquée pour la première fois à propos de Printemps, son deuxième " envoi romain " - dans le rapport peu enthousiaste du secrétaire de l'Académie des beaux-arts à la fin de 1887 -, l'appellation " impressionniste " accolée au nom de Debussy a fait des émules dès son vivant, aidée par des titres évocateurs (comme Reflets dans l'eau, Brouillards, Cloches à travers les feuilles, Et la lune descend sur le temple qui fut, Nocturnes, La mer, Images...) et des techniques d'écriture mettant en valeur les couleurs timbrales et harmoniques ainsi que la dimension la plus insaisissable du temps - l'instant. Voir Fleury 1996. D'ailleurs, Debussy lui-même semble avoir donné l'une des définitions de l'impressionnisme musical les plus justes lorsqu'il écrit à son éditeur Jacques Durand, à propos de ses Images pour orchestre: « Je me persuade de plus en plus que la Musique n'est pas, par son essence, une chose qui puisse se couler dans une forme rigoureuse et traditionnelle. Elle est de couleurs et de temps rythmés » (Debussy 2005, p. 1030).

Pourtant, le compositeur se défendait d'appartenir à ce mouvement. Ainsi, par exemple, dans une interview au Daily Mail du 28 mai 1909, à la question "Vous êtes un impressionniste, M. Debussy? », le compositeur répond : "J'ai été appelé le "Whistler de la musique". [...] Les gens aiment ces noms pompeux. [...] Pour autant qu'il s'agisse de moi, je puis seulement vous dire que mon ambition première, en musique, est d'amener celle-ci à représenter d'aussi près que possible la vie même » (Debussy 1987, p. 293).

En effet, Stefan Jarocinski (1971) considère avec raison que réduire Debussy à l'impressionnisme revient à occulter toute une partie de sa production se rapprochant plutôt du symbolisme. Son goût pour les poètes symbolistes que sont Mallarmé, Verlaine ou Maeterlinck, et pour leurs précurseurs comme Baudelaire et Bourget s'est matérialisé dans un grand nombre d'œuvres, aussi bien vocales qu'instrumentales, jusqu'à son unique opéra achevé, Pelléas et Mélisande, de telle sorte que, dès 1908, M. T. E. Clark souligne les liens qui relient le compositeur à ce courant : «Debussy emploie les accords comme Mallarmé emploie les mots, comme des miroirs qui concentrent la lumière de cent points différents sur le sens exact, mais demeurent des symboles du sens, non le sens lui-même » (Clark cité dans Jarocinski 1971, p. 58-59). 
parce qu'il fait un temps insupportable, préfigurant une autre œuvre citant cet air : Jardins sous la pluie, final du cahier des Estampes.

Si Pierrot est atypique dans la production debussyste, c'est qu'il s'agit d'une œuvre ouvertement comique. Les œuvres citées plus haut font souvent preuve d'un humour plus subtil, moins appuyé. A tel point, que, par exemple, l'inspiration populaire de Jardins sous la pluie passe bien souvent, dans l'oreille des commentateurs, au second plan au profit d'une lecture plus poétique : Harry Halbreich (cité dans Lockspeiser [1962]1989, p. 566) y entend « une stylisation parfaite du bruissement de la pluie, de l'éclaircie et du gazouillis de mille oiseaux frileux et mouillés » tandis que Christian Goubault soutient qu'il s'agit d'une « œuvre brillante, printanière, ruisselante de gouttelettes où joue la lumière »(Goubault 1986, p. 179).

Cette veine d'humoriste du jeune Debussy est maintes fois soulignée dans les différents témoignages contenus dans la revue qui publie cette mélodie pour la première fois. Ainsi, Marguerite Vasnier décrit-elle un Debussy espiègle de 18 ans : "Un jour, des chanteurs des rues s'étaient arrêtés devant la maison; il se met à les accompagner du piano et de la voix, puis leur dit d'entrer et les fait jouer en ajoutant de ces boniments à nous faire mourir de rire » (Vasnier 1926, p. 19).

Paul Vidal, quant à lui, raconte un tour que lui joua son camarade du Conservatoire :

Comme je le harcelais toujours pour qu'il me jouât ses nouvelles compositions, et qu'il n'avait pas le loisir ou la volonté d'en écrire, il me fit cette farce d'apprendre par cœur un recueil de Pessard et il me chantait comme étant de lui Les joyeusetés de bonne compagnie. Je ne découvris que plus tard ce subterfuge (Pierné et Vidal 1926, p. 13).

Que Vidal se laisse berner ainsi par Debussy, en croyant entendre sa musique derrière les notes du recueil de musique légère écrit par Pessard, laisse deviner que l'association de l'humour à Debussy n'est pas, au moins lors de ses jeunes années, artificielle.

Pourtant, si Debussy a publié plusieurs mélodies écrites à cette époque (Pantomime, En sourdine, Mandoline, Clair de lune, Fantoches, Chanson espagnole, Regrets...), il n'inclue pas Pierrot. On ne peut pas supposer que la raison en soit l'utilisation d'une veine comique, qui est présente dans nombre de ces mélodies. On ne peut pas non plus penser qu'il s'agisse du signe d'une œuvre non aboutie, ou dont la qualité d'écriture laisse à désirer. En effet, à son sujet, Charles Koechlin écrit ceci :

Quelques pièces, simplement jolies - fort sages d'ailleurs, - pourraient aussi bien être écrites par tels de ses camarades de classe aux noms oubliés. Il n'en va point de même avec le curieux Pierrot. [...] Voyez, au début, l'harmonisation si caractéristique d'un thème connu. Notez aussi les imitations par quoi se termine le morceau. N'est-ce point déjà l'annonce, et encore mieux, la réalisation partielle de cet humour debussyste que nous aimons dans les Fantoches, dans certains des Préludes, dans les Children's corner et La Boîte à joujoux, et que ce chef-d'œuvre tant souhaité (hélas, jamais écrit), Le Diable 
dans le beffroi aurait affirmé tout entier ${ }^{3}$ ? Ceux qui ont connu Debussy, ce sarcastique sans amertume, et ceux même qui le devinent tel d'après ses articles de la Revue blanche ou du Gil Blas ${ }^{4}$, saisiront ma pensée sans qu'il faille insister davantage. La réalisation du comique, chez Debussy, révèle l'originalité créatrice. [...] Et - même à l'heure actuelle où la plupart se dirigent vers l'humour, croyant parfois l'atteindre en bavardant, sans nul comique musical, sur des poésies à jeux de mots - dites s'il est beaucoup de musiciens dont le sourire se puisse égaler à celui de Claude Debussy (Koechlin 1926, p. 120-121)?

Manifestement, ce n'est pas par manque de qualité que Pierrot ne fit pas partie des mélodies de jeunesse que Debussy choisit de publier. Quelle peut alors en avoir été la raison? Il est possible que cette œuvre qui, à notre connaissance, n'a même jamais été jouée de son vivant, n'était pas destinée à être éditée et portée à la connaissance de tous : elle répondait peut-être davantage à une nécessité intime et personnelle.

\section{Pierrot chez Debussy : Davantage Banville que Margueritte}

Force est de constater que Pierrot balise l'alpha et l'oméga de la vie créatrice de Debussy. Théo Hirsbrunner ne s'y est pas trompé, lui qui ouvre Claude Debussy und seine Zeit avec un chapitre consacré à ce personnage (2002, p. 39-56). Si on le rencontre pour la première fois dans la mélodie qui nous intéresse ici, on le retrouvera bien vite dans Pantomime (1883). Il réapparaîtra à la fin de la vie du compositeur, sans doute à la faveur de la fascination de Debussy pour le Petrouchka de Stravinski ${ }^{5}$, dans La boîte à joujoux (1913), et surtout dans sa Sonate pour violoncelle et piano (1915). En effet, Louis Rosoor, qui a travaillé cette sonate avec le compositeur le 11 octobre 1916 au Moulleau, près d'Arcachon, où Debussy séjourne alors ${ }^{6}$, révéla son intention première qui était d'intituler l'œuvre " Pierrot fâché avec la lune ». Même si cette affirmation

3 Ce « conte musical en 2 actes et 3 tableaux » d'après la nouvelle d'Edgar Allan Poe occupa Debussy, par intermittence, de 1902 à 1911, et était destiné à être créé conjointement avec cet autre opéra d'après Poe qu'est La chute de la Maison Usher, inachevé lui aussi. Le scénario met en scène le Diable, décidé à semer le trouble dans l'esprit étroit des habitants d'un village hollandais, en commençant par sonner treize fois la cloche à midi.

$4 \quad$ Au début du xx $x^{\mathrm{e}}$ siècle, Debussy publia de nombreux articles dans des journaux ou revues en tant que critique musical, qu'il signait du pseudonyme «Monsieur Croche », sans doute en référence à la "Soirée de Monsieur Teste » de Paul Valéry, paru dans Le centaure en 1896. Il y démontrait tous ses talents de plume, en usant d'un ton souvent teinté d'esprit, d'ironie, voire de sarcasme.

5 Le 13 avril 1912, Debussy écrit à Stravinski : « Grâce à vous, j’ai passé d'exquises vacances de Pâques en compagnie de Pétrouchka, du Maure terrible et de la délicieuse Ballerine. J'imagine que vous avez dû passer d'incomparables moments avec ces trois poupées... et je ne connais pas beaucoup de choses qui vaillent ce que vous appelez : "le Tour de Passe-Passe"... il y a là-dedans une sorte de magie sonore, de transformation mystérieuse d'âmes mécaniques qui deviennent humaines par un sortilège, dont jusqu'ici, vous me paraissez l'inventeur unique. Enfin, il y a des sûretés orchestrales que je n'ai rencontrées que dans Parsifal - Vous comprendrez ce que je veux dire, j'en suis sûr! »(Debussy 2005, p. 1503).

6 Comme en témoigne un exemplaire de la partition del'œuvre, dédicacée «À Louis Rosoor./en souvenir d'une audition au Moulleau/et bien cordialement/Claude Debussy/Le Moulleau 11 octobre 1916 ». 
ne repose sur aucune certitude - Debussy ayant manifestement été contrarié par cette révélation ${ }^{7}$, et n'étant pas particulièrement bien disposé à l'égard du violoncelliste ${ }^{8}$ la présence d'une "Sérénade " en guise de mouvement central rend très plausible cette référence à l'univers de la comédie italienne.

Entre ces deux extrêmes de la vie créatrice de Debussy, Pierrot se rencontre à plusieurs reprises en filigrane, comme dans les pièces pour piano Masques et L'isle joyeuse (1903-1904), évoquant l'ambiance des fêtes galantes telles que les peignit Watteau, où les comédiens italiens sont bien souvent à l'honneur. En outre, Pierrot est encore présent dans les projets de ballets inaboutis Masques et bergamasques (1909), et Crimen amoris/Fête galante (1912-1915). Ajoutons à cela les nombreux clairs de lune célébrant l'astre confident de Pierrot, que Debussy a représentés en musique et souvent en association avec la commedia dell'arte, comme dans la Suite bergamasque (1890) ou les deux mélodies sur le même texte extrait des Fêtes galantes de Verlaine (1882 et 1891-1992) ${ }^{9}$. Par ailleurs, selon Hirsbrunner (2002, p. 43), les personnages de Pelléas (ayant encore un pied dans l'enfance) et de Saint-Sébastien (rejetant la convoitise de l'empereur romain) ont la pureté et la naïveté de Pierrot.

Il faut bien l'admettre : le Pierrot que représente Debussy n'est pas en phase avec son époque. En effet, le Pierrot fin-de-siècle s'est laissé contaminer par le décadentisme ambiant et le fumisme des cabarets montmartrois (voir Palacio 1990 et Bonnet 2008). L'année 1882, où Debussy compose sa mélodie, correspond à un moment clé de cristallisation des énergies artistiques autour d'un personnage aux contours renouvelés. En effet, c'est l'année où Jules Laforgue - que Debussy admire au point de parler de lui comme " notre Jules » (Debussy 2005, p. 1972 et 2033) -, après avoir découvert en 1881 le Pierrot sceptique féroce et plein de vices de Hennique et Huysmans $(1881)^{10}$, écrit Pierrot fumiste (Laforgues [1882]2008, p. 71-88).

$7 \quad$ C'est ce qu'affirme Janelle Suzanne Ragno (2005), dans sa thèse de doctorat consacrée à cette œuvre, qui préfère y voir des références au choral luthérien « Ein feste Burg ».

8 La manière dont il décrit le violoncelliste à son éditeur Jacques Durand laisse à penser qu'il semblait peu enclin à la confidence : "Il m'a fait regretter - pendant un moment, d'avoir écrit une Sonate pour violoncelle, et, douter de la sûreté de mon écriture! Enfin, ne "doutons" plus qu'il n'y ait de mauvais musiciens partout! Eh! bien! cette aventure m'a profondément troublé : elle est pleine de conséquences, et je ne m'étonne plus de la fréquente incompréhension qui accueille ma pauvre musique » (Debussy 2005, p. 2036).

9 Sans oublier La terrasse des audiences du clair de lune, tiré du $2^{\mathrm{e}}$ livre des Préludes pour piano (1912), dont l'inspiration ne semble pas être liée à Pierrot, mais plutôt à la ville ruinée d'Amber, en Inde. Voir Lassauzet 2014, p. 29-30.

10 Si Hennique et Huysmans inspirent Laforgue avec cette pantomime, ils subissent quant à eux l'influence conjointe de Zola, en écrivant une "pantomime naturaliste ", et de la troupe anglaise des Hanlon-Lee qui donnait, dans ses numéros joués à Paris depuis les années 1870, une idée plus acrobatique et plus immorale de Pierrot que ne le pratiquaient les Français de l'époque. 


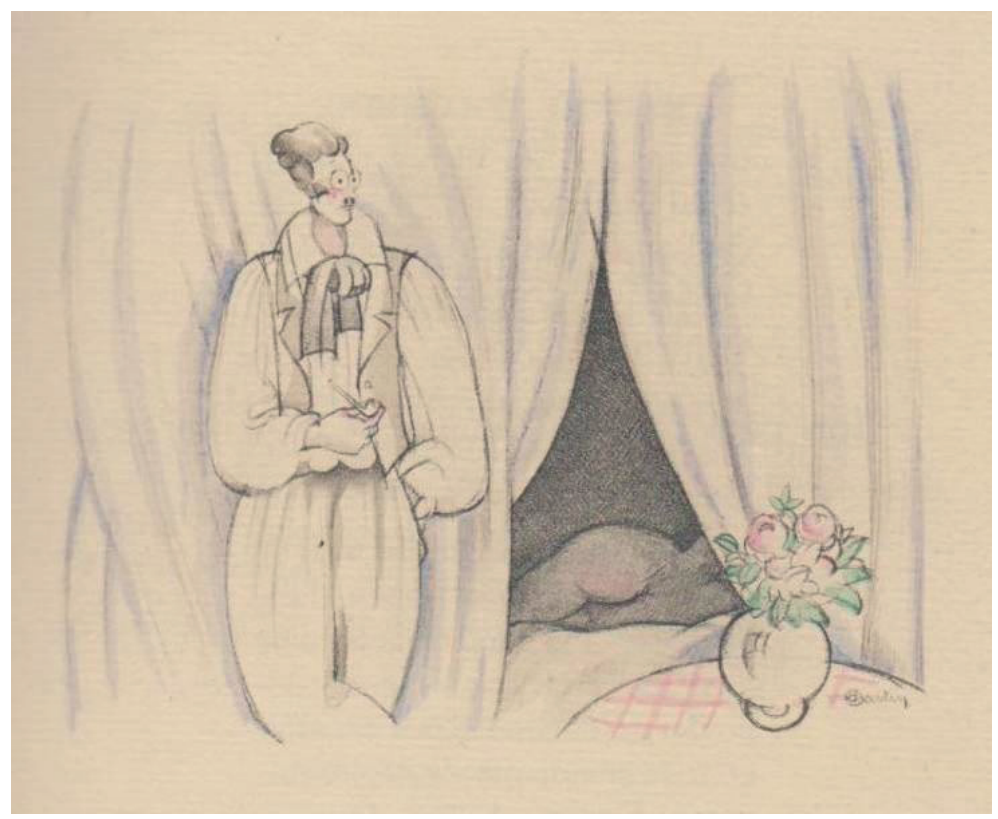

Figure 1: Charles Martin, "Pierrot fumiste", gravure illustrant l'ouvrage de Jules Laforgue.

Il tire son titre de planches parues dans Le chat noir, dessinées par Adolphe Willette (1882), qui publiera quelques années plus tard Pauvre Pierrot (1887), anthologie des centaines de pierrots réalisés par l'illustrateur. Ce dernier nous montre le personnage ivre, adepte des jeux d'argent, vagabond, pendu ou dansant à l'arrivée du choléra. Parfois (c'est un signe des bouleversements qui s'opèrent dans son âme), le personnage de Willette troque son éternelle blouse blanche couleur de lune pour une redingote noire comme la nuit et la mort.

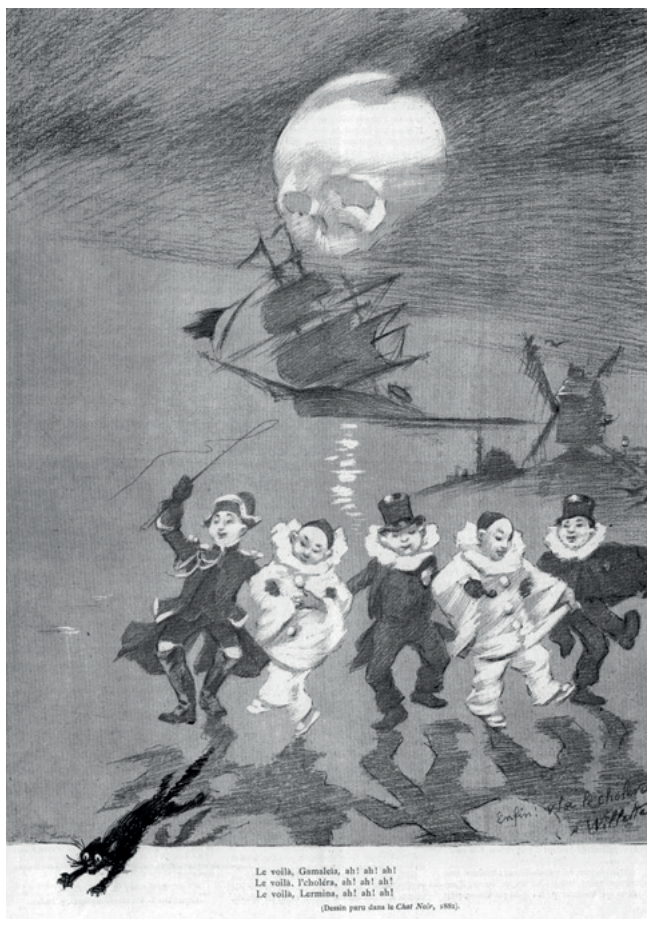

Figure 2: Adolphe Willette, "Enfin, voilà le choléra ", paru dans Le Chat noir en 1882. 
En cette même année 1882, Félicien Champsaur (1896) rédige Pierrot et sa conscience, où, selon son intention, on peut " entendre le battement d'un cœur blessé », et Paul Margueritte présente sa pantomime - qui sera plus tard accompagnée de la musique du camarade de Conservatoire de Debussy, Paul Vidal (Marguerite 1893) Pierrot assassin de sa femme, où se sachant trompé par Colombine, Pierrot la chatouille jusqu'à lui faire rendre l'âme (Marguerite 1882). Comme chez Debussy, «Au clair de la lune » sera utilisé comme un leitmotiv destiné à représenter Pierrot.

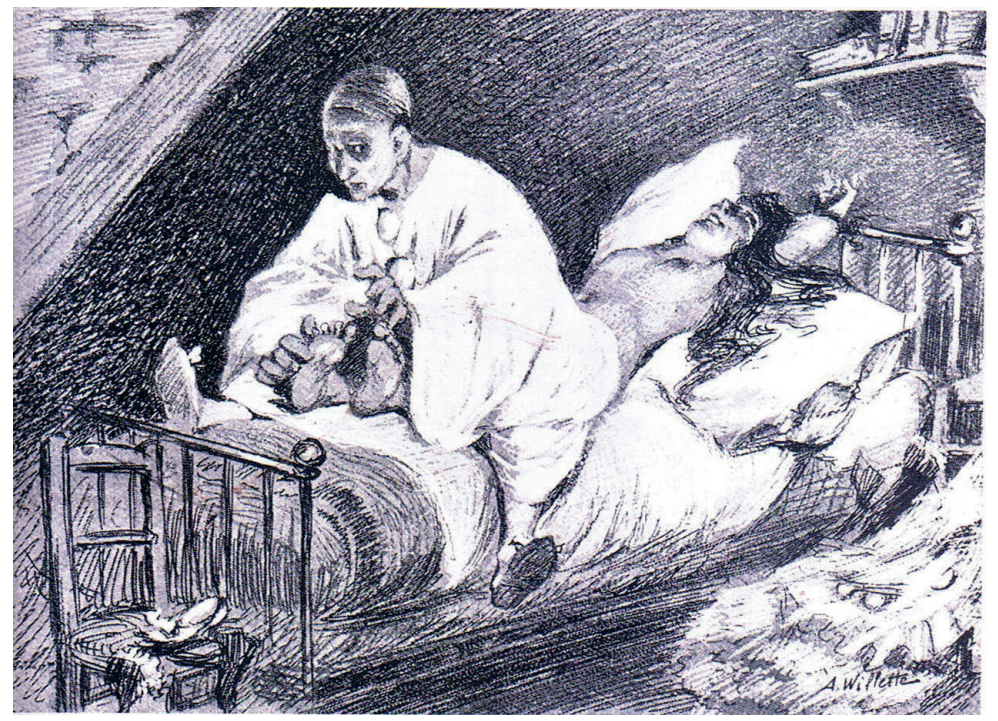

Figure 3 : Adolphe Willette, "Pierrot assassin de sa femme", paru dans Le Pierrot du 7 décembre 1888.

\section{Scène 1}

Pierrot et le Croque-mort
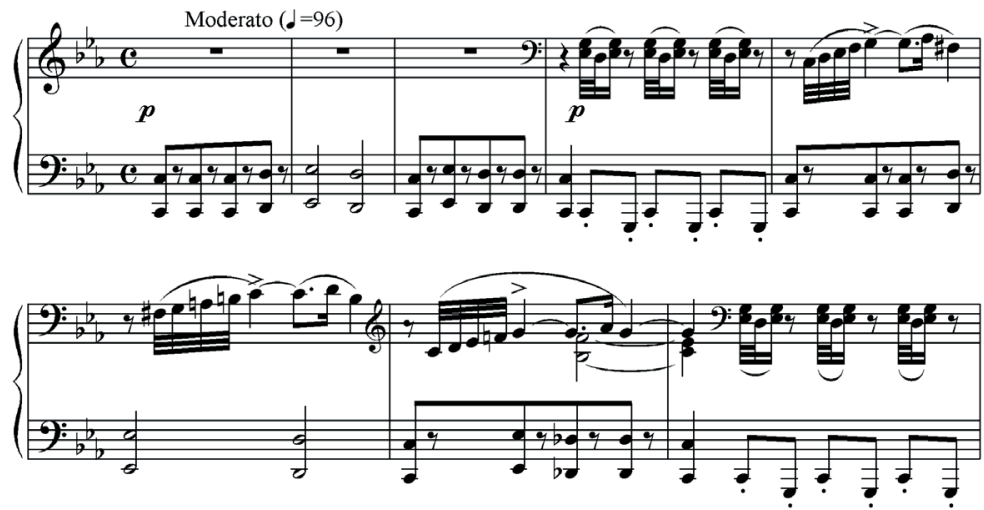

Figure 4: Paul Vidal, Pierrot assassin de sa femme, début de la $1^{r e}$ scène: "Au clair de la lune".

Mais, voleur, meurtrier ou suicidé, le Pierrot fin-de-siècle ne correspond pas aux aspirations de Debussy, qui, en tant que lecteur assidu de Théodore de Banville (Souffrin 1960, p. 200-222), se réfère plutôt à une tradition plus lointaine remontant à la première moitié du xixe siècle, qu'il n'a pas connue, mais qu'il a fait revivre à travers le poème Pierrot, publié en 1842 dans le recueil des Caprices en dizains à la manière de Clément Marot (Banville 1864, p. 181) : 
Le bon Pierrot, que la foule contemple, Ayant fini les noces d'Arlequin, Suit en songeant le boulevard du Temple. Une fillette au souple casaquin

En vain l'agace avec son œil coquin;

Et cependant mystérieuse et lisse

Faisant de lui sa plus chère délice,

La blanche Lune aux cornes de taureau

Jette un regard de son œil en coulisse

À son ami Jean Gaspard Deburau.

Si c'est un Pierrot glouton que Debussy brosse dans Pantomime d'après Verlaine ("Pierrot, qui n'a rien d'un Clitandre/Vide un flacon sans plus attendre/Et pratique, entame un pâté »), ici, c'est l'amoureux qui est à l'honneur. En la matière, parmi les personnages de la commedia dell'arte, Pierrot est l'éternel perdant d'un trio amoureux l'associant à Colombine et à Arlequin. Ainsi, si dans le poème de Banville, Debussy a participé aux "noces d'Arlequin ", c'est certainement pour y constater avec dépit la victoire de son rival sur le cœur de Colombine - d'où l'indifférence de ce personnage sentimental aux avances d'une "fillette au souple casaquin ». Double et projection de la lune, il transforme le croissant de l'astre en " cornes de taureau » du cocu. Si l'amertume se lit en filigrane dans ce poème, elle est sans conséquence : avant que les Décadents ne s'en emparent, Pierrot est un passif, rêveur et mélancolique, pâle et solitaire. C'est ainsi que le représente Banville.

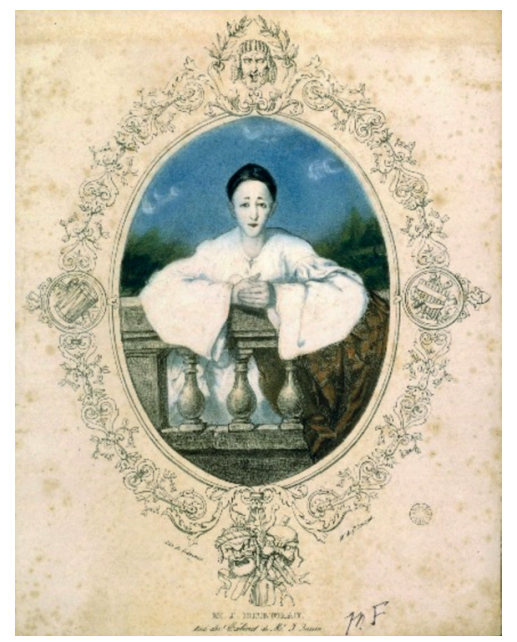

Figure 5:Auguste Bouquet, Jean-

Gaspard-Baptiste Deburau, v. 1830.

En plus de souligner les traits du Pierrot du début du XIX ${ }^{\mathrm{e}}$ siècle, le poème fait référence au monde de la scène où celui-ci se rencontre. En effet, au «boulevard du Temple », est fondé en 1816 le Théâtre des Funambules, où se produira notamment le mime "Jean-Gaspard Deburau », qui sera pour le public parisien, le plus célèbre des pierrots, l'incarnation du personnage sur scène ${ }^{11}$.

11 Le public parisien associe Deburau à Pierrot à tel point que cela conduit à un drame. En effet, 


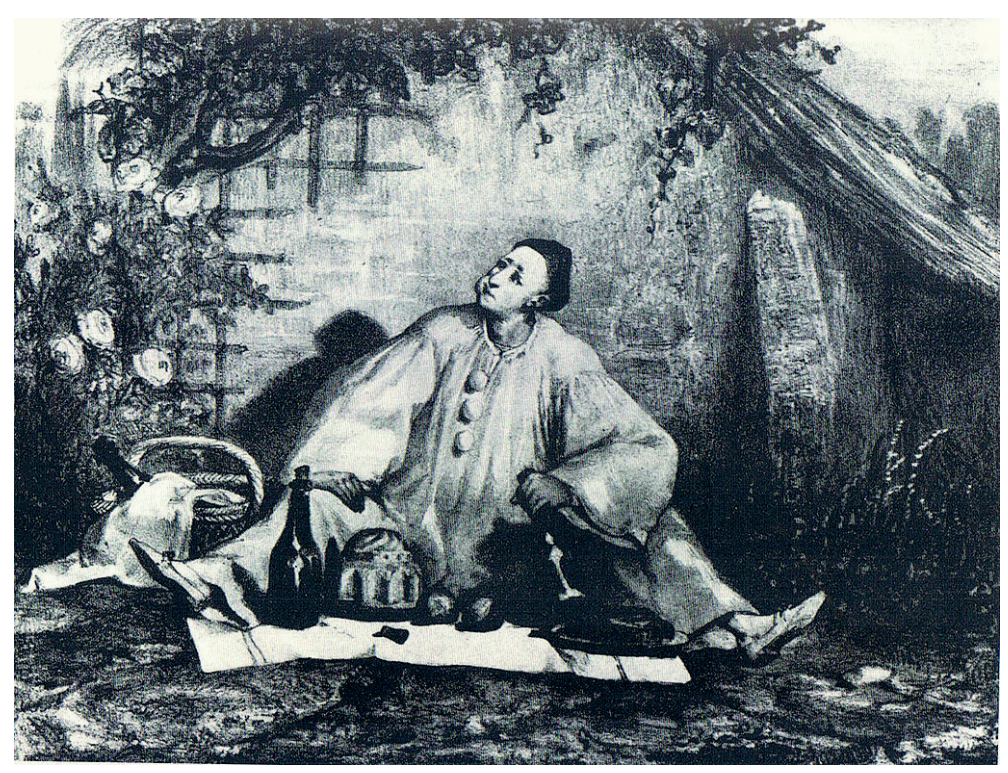

Figure 6: Auguste Bouquet, Le repas de Pierrot (Jean-Gaspard Deburau en Pierrot gourmand), v. 1830.

Cette identification Pierrot/Deburau est telle que, dans le poème, l'identité du protagoniste est ambigüe : celui qui descend le boulevard du Temple devrait être le mime, tandis que la lune devrait en toute logique jeter une oillade à « son ami Pierrot».

\section{Le SYNDROME DE PierRot}

Il faut bien reconnaître que cette identification de l'artiste à son rôle, bien éloignée des conceptions diderotiennes du jeu du comédien (voir Diderot [1930]1994), est une tendance qu'attire ce personnage. En effet, Pierrot représente communément la figure du poète avec tout ce qu'elle implique d'inadaptation et de désuétude, de marginalité et d'anachronisme.

alors qu'il se promenait avec sa femme, Deburau est pris à partie par un garçon, qui s'entête à l'appeler " Pierrot ». Il se saisit alors de sa lourde canne et le frappe si fort que le garçon en perd la vie. Son procès, attirant par centaines des curieux avides d'entendre pour la première fois la voix du mime, aboutit à son acquittement. 


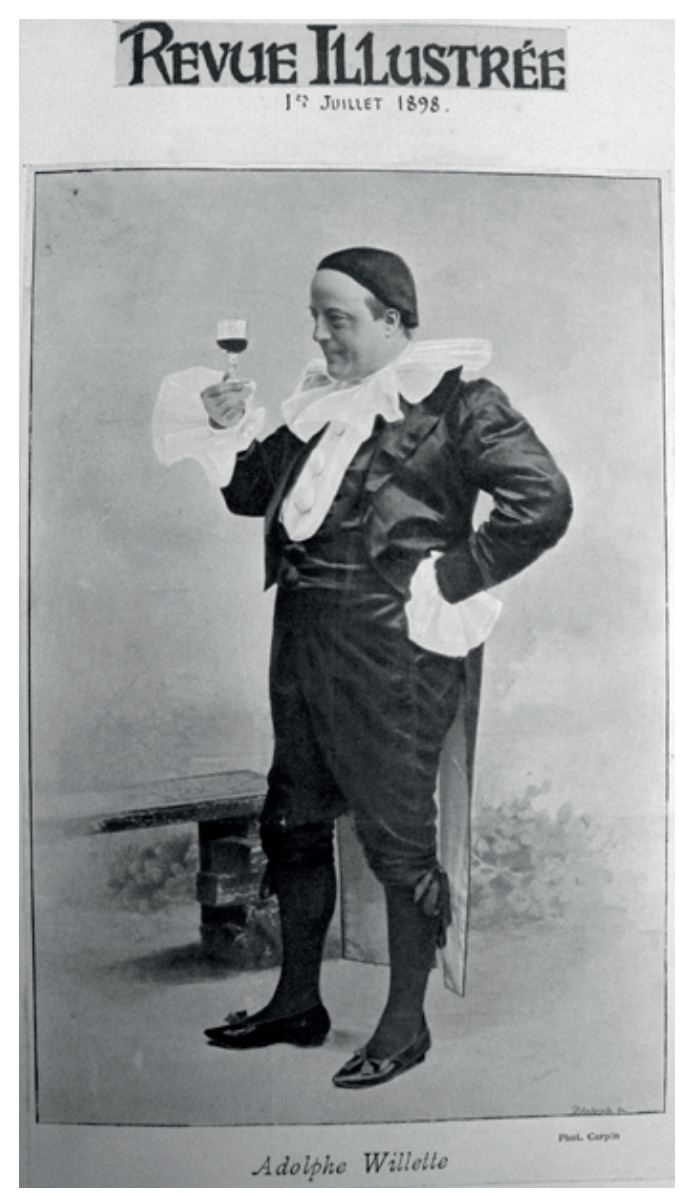

Figure 7 : Adolphe Willette en Pierrot noir en couverture de La Revue illustrée $d u$ $1^{\text {er }}$ juillet 1898.

Ainsi, si Deburau a souvent été considéré comme l'incarnation de Pierrot, il en va de même de plusieurs autres artistes ayant succombé à son charme. Par exemple, lorsque Marcellin Desboutins dessine le portrait de Willette en 1896, c'est dans le costume d'un pierrot noir qu'il le représente - le personnage lui collant tellement à la peau. En juillet 1898, le dessinateur réitère, en se faisant photographier en Pierrot pour La Revue illustrée.

De même, pour ses contemporains, à force de chanter les louanges du "bon Pierrot », Théodore de Banville a fini par se confondre avec lui ${ }^{12}$. Il est par exemple représenté par Catulle Mendès en " Pierrot apollonide " sous le pseudonyme de Déodat de Charmeville (Mendès 1894, p. 341-342). 


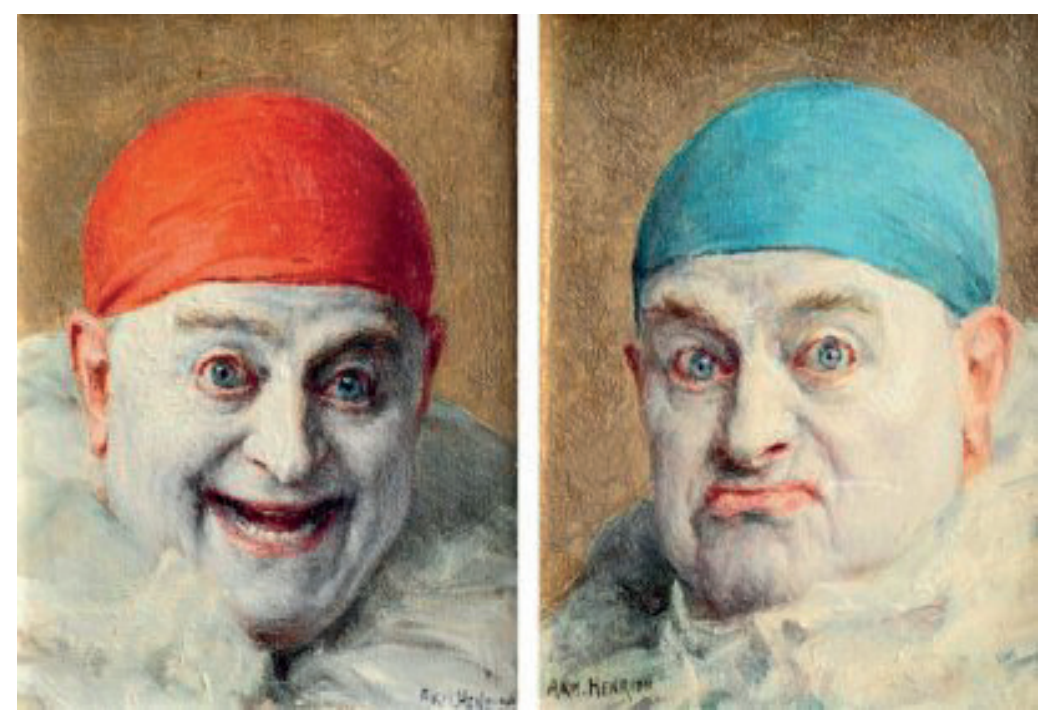

Figure 8: Armand Henrion, Autoportrait au Pierrot content et Autoportrait au Pierrot mécontent. Deux huiles sur panneau, $18 \times 14 \mathrm{~cm}$.

En outre, certains peintres du tournant du siècle ont cédé à la tentation de l'autoportrait en Pierrot, comme Georges Rouault, Amedeo Modigliani, Zinaida Evguenievna Serebriakova ou Armand Henrion ${ }^{13}$. D'ailleurs, comme le souligne Michel Beaujour, l'univers métaphorique du théâtre, auquel appartient Pierrot, est régulièrement convoqué par les auteurs se livrant à d'explicites autoportraits (Beaujour 1980, p. 292-293). Ainsi, par exemple, Barthes explique : "L'effort vital de [Roland Barthes par Roland Barthes] est de mettre en scène un imaginaire : "Mettre en scène" veut dire : échelonner des portants, disperser des rôles, établir des niveaux et, à la limite : faire de la rampe une barre incertaine " (Barthes 1975, p. 109).

La musique étant l'art qui lui est le plus généralement associé - le personnage jouant volontiers la sérénade à Colombine et étant le seul de la commedia dell'arte à être associé à une chanson -, on rencontre d'autres identifications, de musiciens cette fois, au personnage. Ainsi, on a pu considérer que Schoenberg projetait ses frustrations artistiques dans son Pierrot lunaire (1912), et notamment dans la «Serenade " ( $\left.\mathrm{n}^{\circ} 19\right)$. Ce mélodrame met en scène Pierrot qui, chantant devant le balcon de sa belle accompagné du violoncelle, et ne recevant que les critiques de son tuteur Cassandre, décide face à ce désaccord stylistique manifeste de jouer avec son archet géant sur le crâne de ce dernier. D'aucuns ont pu voir, cachés derrière le personnage antipathique de Cassandre, les critiques qui menaient la vie dure au compositeur avant-guardiste, qui aurait ainsi décidé de se venger en musique, à la fois dans cette pièce et dans "Gemeinheit! » ( $\left.\mathrm{n}^{\circ} 16\right)$, où Pierrot creuse, toujours au violoncelle (l'instrument de Schoenberg), dans le crâne d'un Cassandre hurlant de douleur pour y fixer une pipe (voir Simms 2000, p. 129-130 et Buch 2006, p. 218-223).

13 Pendant ce temps, Picasso préfère quant à lui se représenter sous les traits d'Arlequin. Pour une étude approfondie de l'identification des artistes aux personnages de la commedia dell'arte et autres clowns, voir Starobinski (1970). 


\section{DeBussy SUCCOMBE}

Debussy aurait-il lui aussi été tenté de s'identifier à Pierrot? Cette hypothèse pourrait s'appuyer sur la récurrence du personnage dans la production du compositeur. Ainsi, Dominique Jameux a-t-il pu, à la faveur d'une double association de Pierrot à l'Artiste (donc au compositeur), et de la Lune à la Poésie (donc à la musique), avancer que le sous-titre originel de la Sonate pour violoncelle, "Pierrot fâché avec la lune ", cacherait la frustration de Debussy à l'égard de la musique, lui échappant à la fin de sa vie (Jameux 2002, p. 283) ${ }^{14}$.

De nombreux indices tendent à corroborer la thèse d'une identification de l'artiste à son modèle. D'abord, Marguerite Vasnier se souvient que le jeune Debussy aimait à imiter l'amoureux chantant devant le balcon de Colombine : "Faisant de sa canne une guitare, [il] prenait la pose du chanteur florentin et improvisait des chansonnettes et des sérénades ou parodiait la musique italienne qu'il n'aimait pas » (Vasnier 1926, p. 19). Debussy lui-même comparera sa condition d'artiste à celle du rêveur lunaire, dans une lettre de 1904 à sa femme Lilly : " En somme, entre un chirurgien et un artiste la balance ne se fait pas! C'est d'ailleurs assez juste... le premier sert à quelque chose de défini, c'est un homme officiel et public, qui n'habite pas la lune! Tandis que le second! rêveur, maussade quelquefois, il ne sert proprement à rien, qu'à amuser quand il le peut - ses semblables » (Debussy 1890, p. 185-188).

Théo Hirsbrunner souligne également que, dans ses jeunes années, comme le Pierrot fin-de-siècle, Debussy est tout autant bohème - fréquentant les bars et cabarets montmartrois, comme le Chat noir où officie Willette, qui illustrera la partition de Mandoline en 1890 pour La Revue illustrée (p. 185-188) - que dandy ayant le goût des belles et bonnes choses, bien que sans le sou, collectionnant des objets d'art en tout genre et cultivant l'élégance (Hirsbrunner 2002, p. 46).

14 À demi-mots, l'idée avait déjà été suggérée en 1939 par W. H. Mellers dans un article consacré au dernier Debussy (Mellers 1939, p. 168-176). 


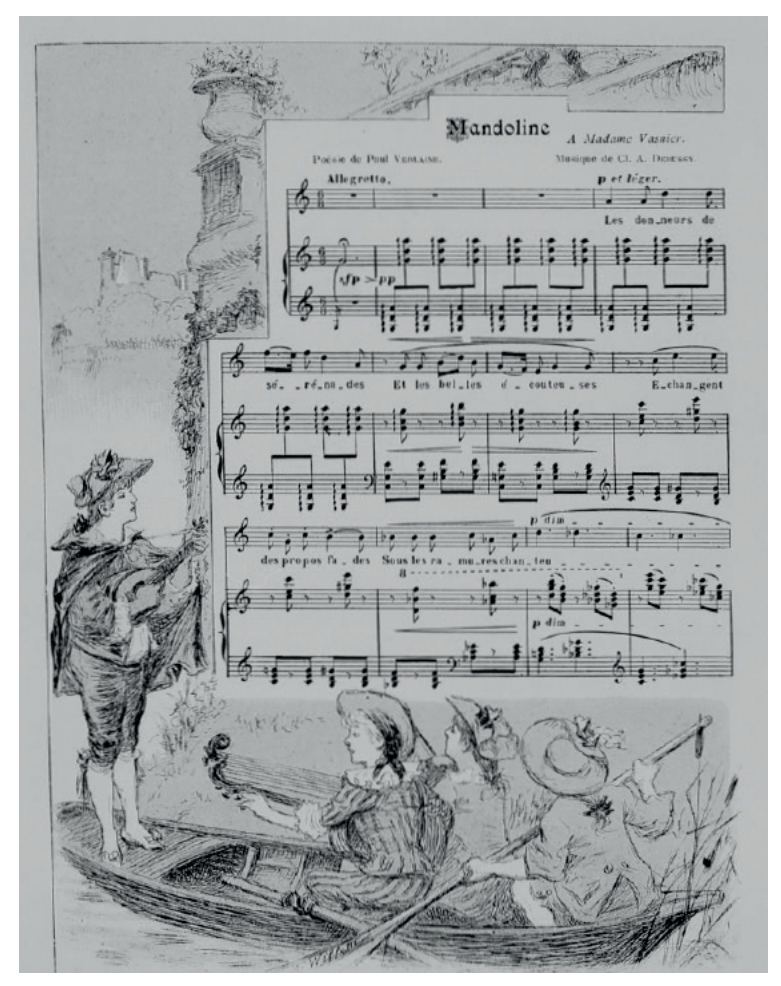

Figure 9 : Claude Debussy, Mandoline, illustré par Willette dans La Revue illustrée du 15 juin 1890.

Mais surtout, nous savons par Edward Lockspeiser que, lorsqu'il fut engagé en tant que pianiste par Mme von Meck durant les étés 1880 à 1882, Debussy était affublé de différents surnoms par la famille, parmi lesquels "le bouillant Achille " (Lockspeiser [1962]1989, p. 67), Bussik, Bussikov... mais aussi Petrouchka c'est-à-dire Pierrot en russe (ibid, p. 69).

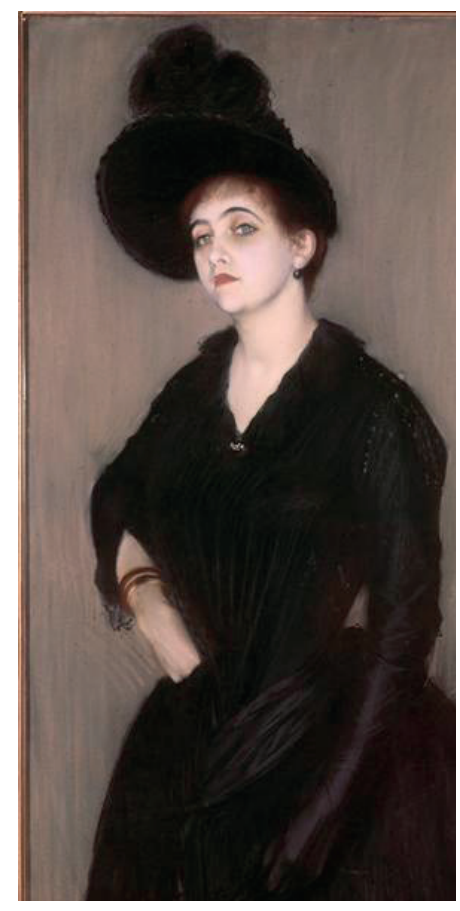

Figure 10: Portrait de Marie-Blanche Vasnier par Jacques Emile Blanche, 1888. Pastel sur carton, $130 \times 66 \mathrm{~cm}$. Paris, Petit Palais, musée des BeauxArts de la Ville de Paris. 
Dans ces conditions, il serait probable de voir dans Pierrot une projection subjective de la part du compositeur. D'autant qu'il semblerait qu'au fil de sa carrière, Debussy ait placé des références personnelles dans ses œuvres. Ainsi, par exemple, les deux mouvements extrêmes de la Sonate pour violon et piano indiquent un tempo de 55 par valeur rythmique (respectivement la blanche pointée et la noire pointée), ce qui correspond à l'âge du compositeur alors qu'il écrit cette ouvre. Aussi semble-t-il mettre en pratique le fameux adage de Cosme de Médicis qui veut que tout peintre se peigne lui-même ("Ogni pittore dipinge sè»).

Mais, si Debussy voulait se représenter derrière le personnage de Pierrot, il avait le choix parmi une grande quantité de poèmes, et n'a, paraît-il, pas opté pour celui-ci par hasard. En effet, Marguerite Vasnier rapporte que «quand il lisait un volume de poésie, le choix d'une pièce à mettre en musique était le sujet de longs débats » (Vasnier 1926, p. 20).

Effectivement, le texte de Banville, qui comporte un rapport ambigu entre la scène et la vie réelle, suggère cette projection de l'artiste dans la peau du personnage, et nous pensons que cet élément pourrait avoir guidé son choix. Mais c'est le sujet principal du poème qui nous semble déterminant : l'histoire d'amour malheureuse de Pierrot.

En effet, Pierrot est dédié " à Madame Vasnier ». Marie-Blanche Vasnier, qu'il rencontre alors qu'il exerce l'activité d'accompagnateur pour le cours de chant de Madame Moreau-Sainti, est de douze ans son aînée, mariée à un greffier des bâtiments, et a deux enfants (dont Marguerite). Le jeune Debussy est rapidement séduit par la voix de cette soprano léger, dont la plasticité lui doit de devenir sa muse : entre 1880 et 1884, il écrit une trentaine de mélodies qui lui sont destinées ${ }^{15}$. En échange, elle l'accueille volontiers dans sa famille, comme un second foyer où le musicien jouit d'un climat favorable à son épanouissement intellectuel et culturel. De plus, il bénéficie de l'amitié et du support financier de son époux Henri Vasnier.

Mais à la teneur des poèmes choisis, souvent amoureux, on comprend sans peine que Debussy n'était pas uniquement intéressé par la voix de Mme Vasnier, comme en témoignent les dédicaces accompagnant les partitions de ces pièces. Dès la première, Caprice, on peut lire - bien que biffé, peut-être pour éviter d'éveiller les soupçons du mari : "ces mélodies conçues en quelque sorte par votre souvenir ne peuvent que vous appartenir comme vous appartient l'auteur » (Lesure 1962, p. 22); dans un recueil regroupant plusieurs des mélodies composées pour elle, la dédicace dit « à Madame Vasnier/ces chansons qui n'ont jamais vécu que par elle et qui perdront leur grâce charmeresse si jamais plus elles ne passent par sa bouche de fée mélodieuse./L'auteur éternellement reconnaissant » (ibid., p. 23). La dédicace de Tragédie lève toute équivoque : "À Madame Vasnier, la seule muse qui m'ait inspiré quelque chose ressemblant à un sentiment musical (pour ne parler que de celui-là)» (Debussy cité dans Fulcher 2001, p. 102).

15 On trouve, parmi ces mélodies, Caprice, Les baisers, Rondel chinois, Tragédie, Jane, La fille aux cheveux de lin, Les papillons, Fantoches, Les roses, Sérénade, Pierrot, Fête galante, Chanson des brises, Flots, palmes, sables, Ode bachique, En sourdine, Mandoline, Séguidille, Clair de lune, Pantomime, Chanson espagnole, Coquetterie posthume, Romance ("Voici que le printemps"), Mélodie, Paysage sentimental, Apparition, Romance ("Silence ineffable de l'heure "), La romance d'Ariel et Regret. 


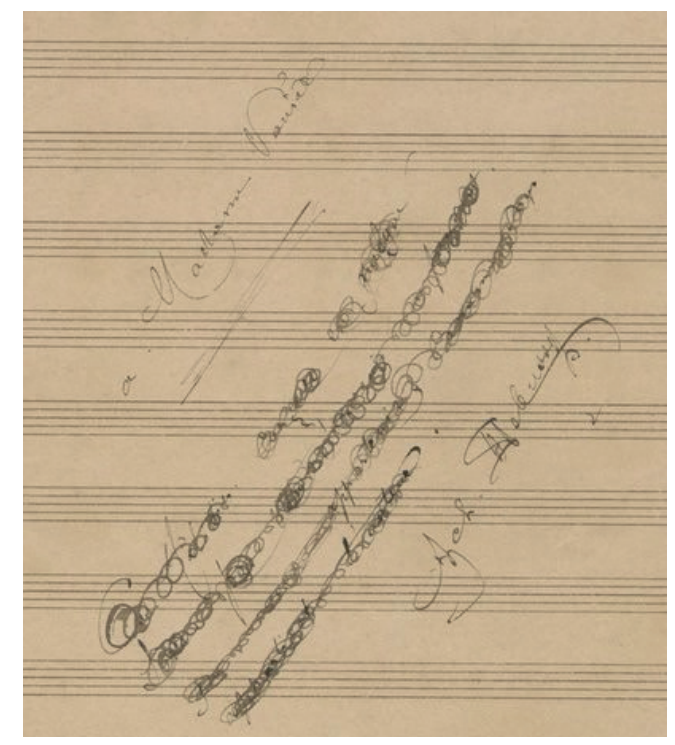

Figure 11 : Dédicace de Debussy à Madame Vasnier sur la page titre de Caprice (manuscrit autographe).

Mais, quand Debussy tente et remporte le prix de Rome en 1884, leur relation est interrompue, le compositeur étant contraint de s'exiler dans la cité éternelle. Alors qu'il s'apprête à rentrer à Paris pour quelques mois en juillet 1885, il reçoit une lettre de Mme Vasnier qui le met au désespoir, comme en témoignent ces lignes qu'il écrit à son ami Gustave Popelin :

La dernière lettre d'elle que j'ai reçue avant-hier, cachait mal tout l'ennui que lui donnerait ma présence là-bas. Me disant qu'il serait très imprudent de nous voir, tu comprends que souffrance pour souffrance, j'aime mieux rester ici que de m'exposer aux rages folles que me donneront sûrement ces empêchements de la voir, tout en étant près d'elle, cette vie serait insupportable avec la jalousie que je me connais, la forcer à faire autrement serait la perdre, eh bien j'aime encore mieux la perdre, en gardant l'orgueil de mon amour, que de jouer ce rôle de chien suppliant, qu'on finit par laisser à la porte, je lui ai dit du reste, que je ne voulais rien de changé, et qu'elle soit toute à moi (Debussy 2005, p. 34).

Ce premier amour de Debussy est donc un amour impossible : Madame Vasnier est mariée et bien peu encline à quitter son époux, au grand dam du jeune artiste. De ce fait, celui-ci, dont nous avons souligné plus haut l'inclination pour Pierrot, pourrait avoir trouvé dans le triangle amoureux dans lequel est engagé le personnage avec Colombine et Arlequin un éloquent parallèle avec sa propre situation. Ainsi, il est frappant qu'aient été conjointement écrites deux mélodies d'après Banville sur la même thématique : Sérénade ("Las! Colombine a fermé le volet,/Et vainement le chasseur tend ses toiles, /Car la fillette au doux esprit follet,/De ses rideaux laissant tomber les voiles,/S'est dérobée, ainsi que les étoiles ») et Pierrot, dans lequel le personnage quitte " les noces d'Arlequin ». Debussy, sous les traits du personnage lunaire, serait donc le perdant - le cour de Colombine-Madame Vasnier appartenant 


\section{à Arlequin-Henri Vasnier.}

Bien que l'histoire amoureuse du jeune Debussy et l'ambigüité entre le personnage et l'acteur suggérée par le texte invite à une lecture autobiographique de cette mélodie, nous en sommes pour l'instant à l'état d'hypothèse. Il s'agirait, pour la confirmer, de trouver dans la musique de quoi abonder dans ce sens.

\section{LA PREUVE DANS LA MUSIQUE}

\section{L'autoportrait musical à l'allemande}

Lorsqu'un compositeur souhaite indiquer sa présence dans ses œuvres, voire dresser son autoportrait, le moyen le plus commode est d'utiliser l'alphabet musical. En effet, la désignation des notes allemande - tout comme l'anglo-saxonne -, passe par des lettres, en commençant par la (A), ce qui permet de se livrer à une transposition sonore des noms. Ainsi, «Bach » donne un motif à quatre notes : si bémol (B) - la $(\mathrm{A})-d o(\mathrm{C})-$ si bécarre $(\mathrm{H})$. Le compositeur l'utilise par exemple à la fin de l'Art de la fugue (BWV 1080).

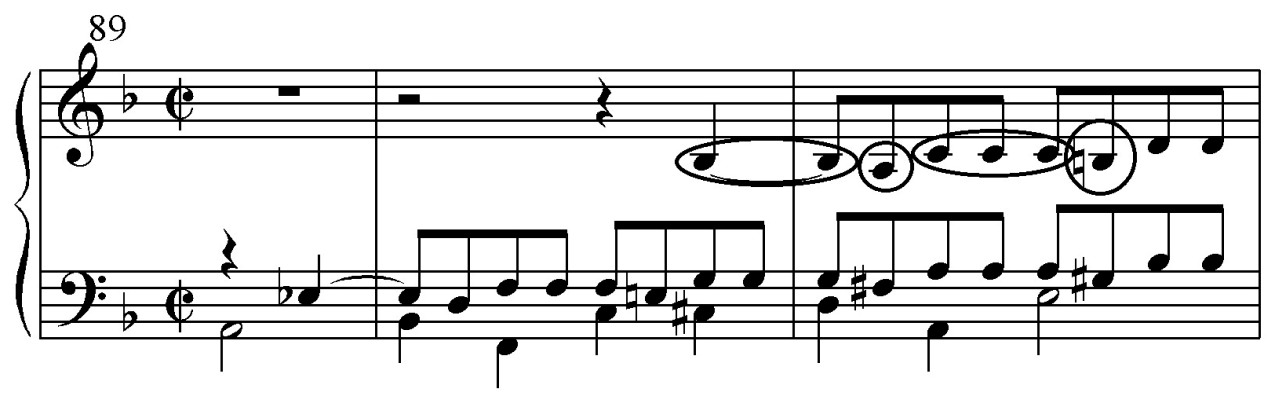

Figure 12 : Jean-Sébastien Bach, Die Kunst der Fuge, Contrapunctus 11 a 4, mes. 89-91 : le motif $B-A-C-H$.

Schumann, aussi féru de ce procédé que son aîné, cite ce motif dans les Fugues pour orgue, piano ou harmonium, op. 60. Il transforme également en notes de musique son propre nom dans Carnaval, op. 9 : ne gardant que les notes appartenant à l'alphabet musical, il obtient mi bémol $(\mathrm{S})-d o(\mathrm{C})$ - si bécarre $(\mathrm{H})-l a(\mathrm{~A})$, motif du premier des Sphinx situés au centre du cycle.

$\mathrm{Au} \mathrm{xx}^{\mathrm{e}}$ siècle, Alban Berg, dans son Kammerkonzert, ouvre son premier mouvement par un motif de cinq mesures où sont représentés les trois représentants de la Seconde Ecole de Vienne, successivement Arnold Schoenberg, à qui l'œuvre est dédiée, au piano (AD soit la-ré, et SCHBEG, mi bémol-do-si-si bémol-mi-sol), Anton Webern au violon (A soit la, puis EBE soit mi-si bémol-mi) et enfin le compositeur lui-même au cor (ABA soit la-si bémol-la, BEG soit si bémol-mi-sol). 


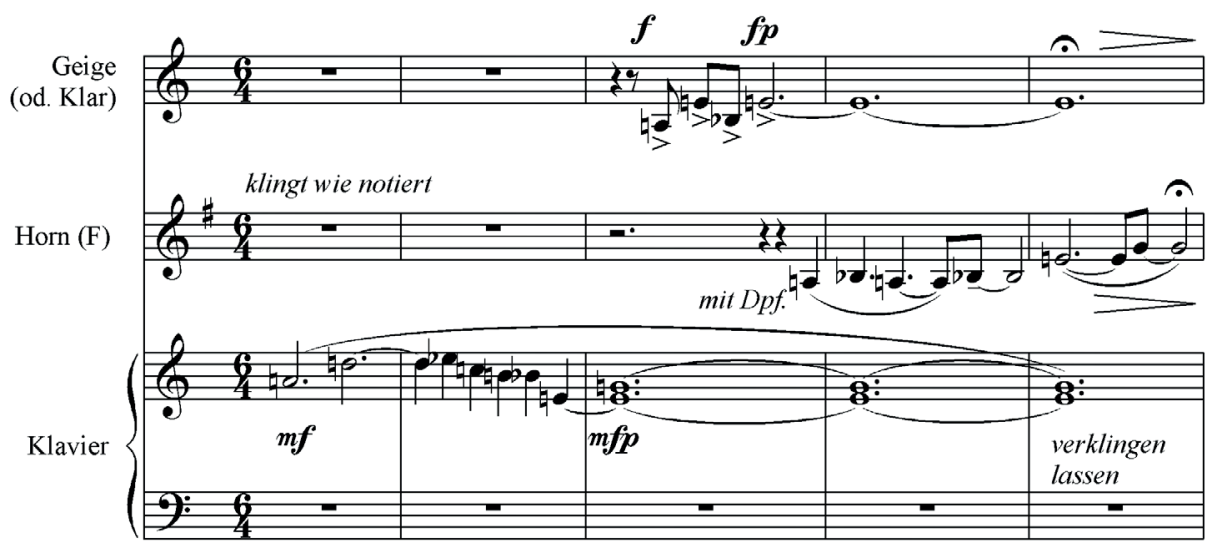

Figure 13 : Alban Berg, Kammerkonzert, I. Tema scherzoso con variazoni, mes. 1-5: $A D-S C H B E G$ (piano), A-EBE (violon), $A B A-B E G$ (cor).

On remarquera sans peine que ce procédé est davantage prisé des compositeurs germaniques, ayant l'habitude de désigner les notes par des lettres. Les Français y sont moins sensibles, et si Debussy l'utilisera en 1909 dans son Hommage à Haydn (à partir du motif si-la-ré-ré-sol ${ }^{16}$ ), ce ne sera que suite à la demande de la revue S.I.M. en vue de la publication d'un numéro spécial célébrant son anniversaire.

En effet, les musiciens français ne désignant pas les notes par des lettres, il leur a fallu trouver d'autres moyens de se présenter en musique, plus en phase avec le système hérité de Guy d'Arezzo. Par chance, notre compositeur possède un nom transposable en son, puisque la dernière syllabe de son nom (-sy) rappelle la note si. Reste à savoir si le musicien a saisi l'opportunité de se représenter de cette manière...

\section{L'autoportrait musical à la Debussy}

Dans Pierrot, la mise en musique du dernier vers est parée de mystère puisqu'elle fait totalement disparaître la mélodie "Au clair de la lune », qui était répétée sans cesse jusqu'ici. Pourtant, il s'agit de la seule partie du poème qui devrait exiger l'air

16 Si les Allemands et les Anglo-saxons désignent leurs notes de musique par des lettres, ceci s'arrête à $\mathrm{G}(\mathrm{sol})$, voire $\mathrm{H}$ pour le cas particulier du si, possédant deux formes (H pour le bécarre, B pour le bémol). Au-delà, les lettres ne correspondent plus à des notes. Les éditeurs de la revue S.I.M., afin de transcrire dans son intégralité le nom de Haydn, ont étendu le principe de correspondance à tout l'alphabet, de la manière suivante :

\begin{tabular}{|c|c|c|c|c|c|c|}
\hline La & Si & Do & Ré & Mi & Fa & Sol \\
\hline A & $\begin{array}{c}\text { B } \\
(H)\end{array}$ & C & D & E & F & G \\
\hline H & I & J & K & L & M & N \\
\hline O & P & Q & R & S & T & U \\
\hline V & W & X & Y & Z & & \\
\hline
\end{tabular}


populaire : en effet, "A son ami Jean-Gaspard Deburau » rappelle « Mon ami Pierrot » $\mathrm{du}$ chant populaire, tout en soulignant à nouveau cette mutation de l'artiste en son personnage.

$\mathrm{Si}$ «Au clair de la lune » disparaît temporairement de la trame musicale, c'est que se joue dans le texte un moment clé par rapport à la thématique de la transformation, que Debussy va relayer à son instrument - le piano. Comme le nom "Deburau ", cité dans le poème, nourrit une ressemblance troublante avec "Debussy ", le compositeur investit subjectivement ce poème en remplaçant -rau par-sy, de la manière suivante : lorsque la chanteuse émet la syllabe -rau, le piano, à la faveur d'un accord de mi, joue à la basse sa quinte si. Debussy prend d'ailleurs soin d'anticiper le mi grâce à une petite note afin que le si soit mis en valeur sur le temps. De cette manière, Debu-rau devient Debus-si. A ce moment, réapparaît « Au clair de la lune » en sixtes parallèles, culminant dans sa partie inférieure sur cette même note.

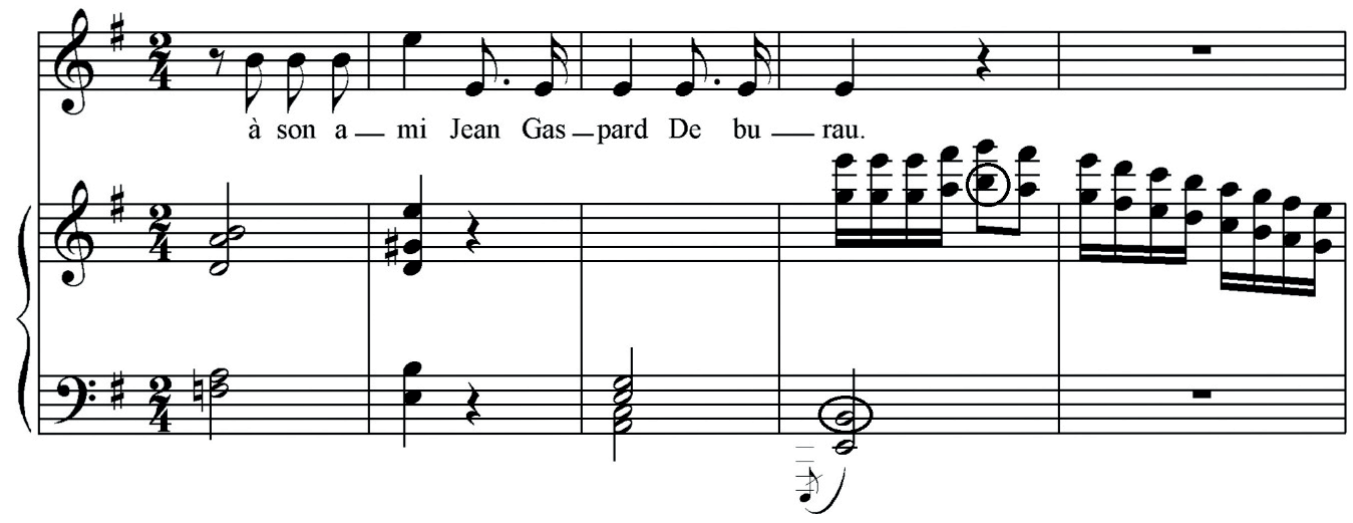

Figure 14 : Claude Debussy, Pierrot, mes. 48-52.

Ceci pourrait sembler fortuit si, au cours de sa vie, Debussy ne s'était pas à plusieurs reprises représenté par cette même note, correspondant à la sonorité de la dernière syllabe de son nom. Par exemple, dans une lettre à Louis Laloy du 25 juin 1909 où il signe en dessinant une simple portée avec une clé de sol et un si (Debussy 2005, p. 1192). On trouve également un mot écrit sur un bout de papier à musique pour son épouse Emma, à l'occasion de Pâques 1912, où Debussy ajoute à sa signature une note bémolisée - sans doute un si à nouveau, la première note de l'ordre des bémols (ibid., p. 1501).

Une phrase énigmatique dans un envoi à André Caplet du 22 juillet 1916 semble aussi aller dans ce sens : "Évidemment le sort du si bémol, triple croche pointée comme ce musicien est vicieux? - n'est pas enviable » (ibid., p. 2009). Dans l'édition de la correspondance du compositeur, il est dit que Caplet, remarquable correcteur, " avait sans doute signalé à Debussy une erreur rythmique dans la Sonate pour violoncelle et piano ». Or, il s'avère qu'il n'y a aucune triple croche pointée dans cette œuvre, encore moins sur si bémol. Si la signification était ailleurs?

Admettant que le si soit la note par laquelle il se représente, c'est son propre sort que Debussy n'estime pas enviable. Il s'agit alors ici davantage d'une plainte de la part d'un compositeur angoissé par la guerre - à laquelle il fait référence par la suite : " vous êtes avec de braves gens qui doivent avoir oublié les petites complications dans 
lesquelles se complaisent les gens qui ne sont sur aucun front »-, et par l'état de santé de sa femme (souffrant de trachéite) et de sa fille (atteinte d'une coqueluche), et surtout éprouvant de terribles douleurs à cause de son cancer. Tout ceci explique à la fois l'adjonction du bémol, symbolisant cette humeur chagrine par l'abaissement de la note, l'aparté (" comme ce musicien est vicieux?») et le rythme alambiqué, rappelant que l'état de celui qui se faisait appeler autrefois Monsieur Croche lorsqu'il écrivait des critiques musicales dans la Revue blanche ou le Gil Blas s'est bien dégradé.

A la lecture de ces différentes lettres, il devient évident que le compositeur se représentait volontiers en musique par un si, rappelant par transformation la dernière syllabe de son nom. Or, il s'avère que cette note, en plus d'apparaître sous le rau de «Deburau », joue un rôle de premier ordre dans Pierrot.

En effet, non contente d'être la première note jouée au piano et la dernière qui soit chantée, sa présence devient criante lorsqu'elle vient perturber la première présentation du thème de Pierrot ("Au clair de la lune ») en do majeur (mesure 2). Considérant, ainsi, que si représente Debussy et do Pierrot, nous constatons que les mesures d'introduction mettent en scène la progressive incarnation du personnage par le musicien.
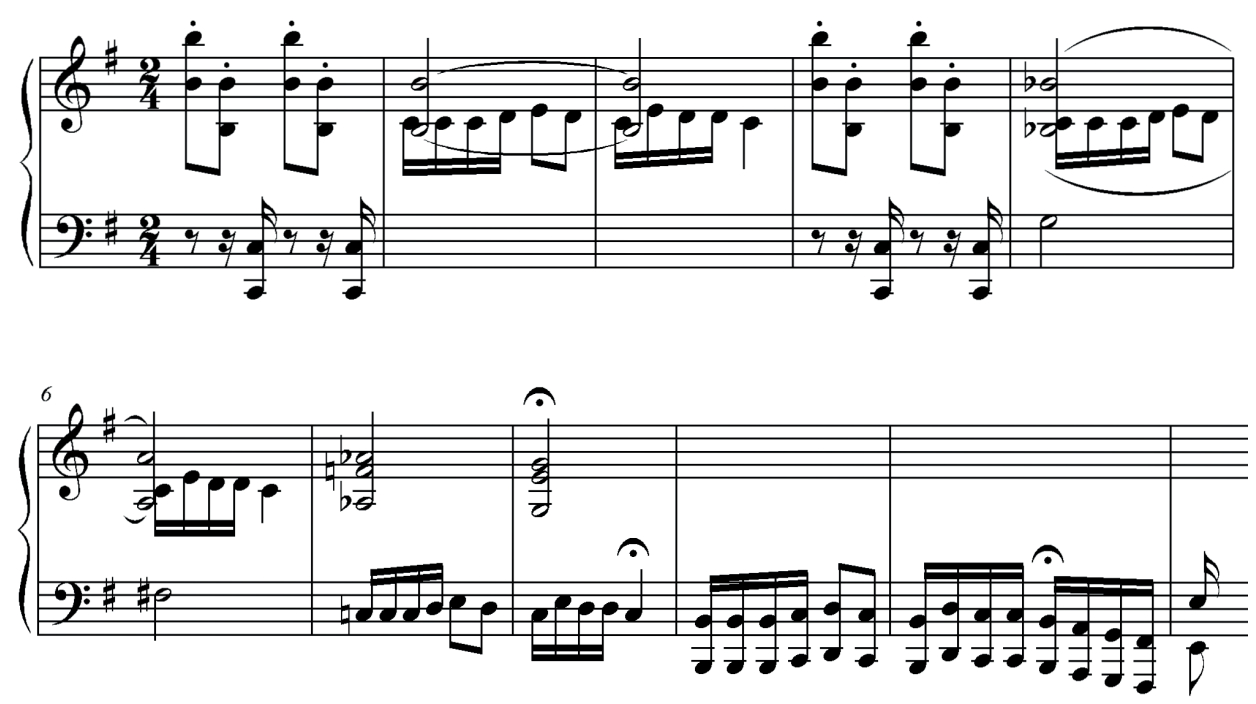

Figure 15 : Claude Debussy, Pierrot, mes. 1-10.

D'abord, Debussy (les si aigus) et Pierrot (les do graves) sont juxtaposés (mesure 1), avant d'être superposés sur un registre similaire, accentuant la dissonance de cette rencontre incongrue (mesure 2). Pour l'heure, les deux entités sont nettement séparées. Mais les mesures suivantes (mesures 5 à 8) règlent l'association malheureuse par un accompagnement harmonique en descente chromatique menant à l'harmonisation correcte de «Au clair de la lune » sur l'accord de do majeur tenu par un point d'orgue.

C'est alors que la transformation a lieu : la mélodie populaire est énoncée, non plus sur do mais si, dans le grave, c'est-à-dire dans la tessiture vocale de Debussy, qui était baryton-basse - comme en témoigne la Petite cantate sur grand papier pour le jour de sa fête écrit en 1907 pour l'anniversaire d'Emma, où un " récitant " (c'est-à-dire lui-même) chante dans cette tessiture (Debussy 2005, p. 1009-1012). Ainsi, l'asso- 
ciation d'une modulation et d'un registre abaissé par rapport à la version originale de la mélodie populaire indiquent que la transformation de Debussy en Pierrot est achevée. Si, en tant que dominante, invite la voix à entrer sur la tonique mi. Or, on retrouve à la mesure 11 la $7^{\mathrm{e}}$ do-si (devenant do-si bémol mesures 13-14). Mais celle-ci est devenue une $2^{\text {de }}$ intégrée dans un accord de tonique à sixte ajoutée : la dissonance est à présent totalement désamorcée, alors que la voix chante "bon pierrot " sur des si, confirmant l'appropriation du personnage par le compositeur.

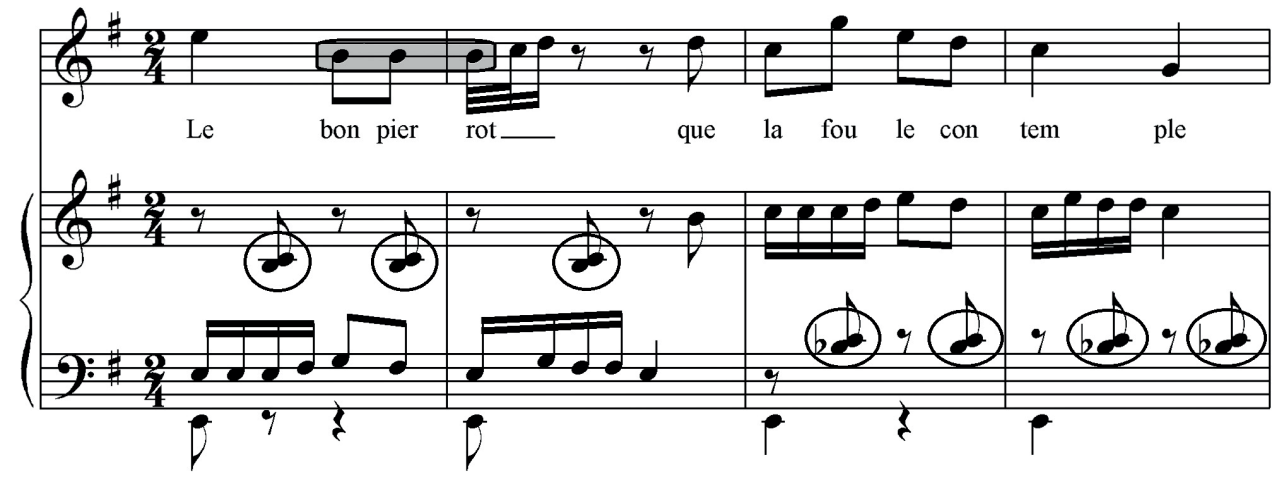

Figure 16: Claude Debussy, Pierrot, mes. 11-14.

Preuve supplémentaire de la projection du compositeur dans le personnage de Pierrot, tout au long de cette mélodie, le pianiste (c'est-à-dire Debussy) sera le seul à jouer le thème de Pierrot ( Au clair de la lune »). Une unique exception se trouve aux mesures 31 et suivantes à la voix :

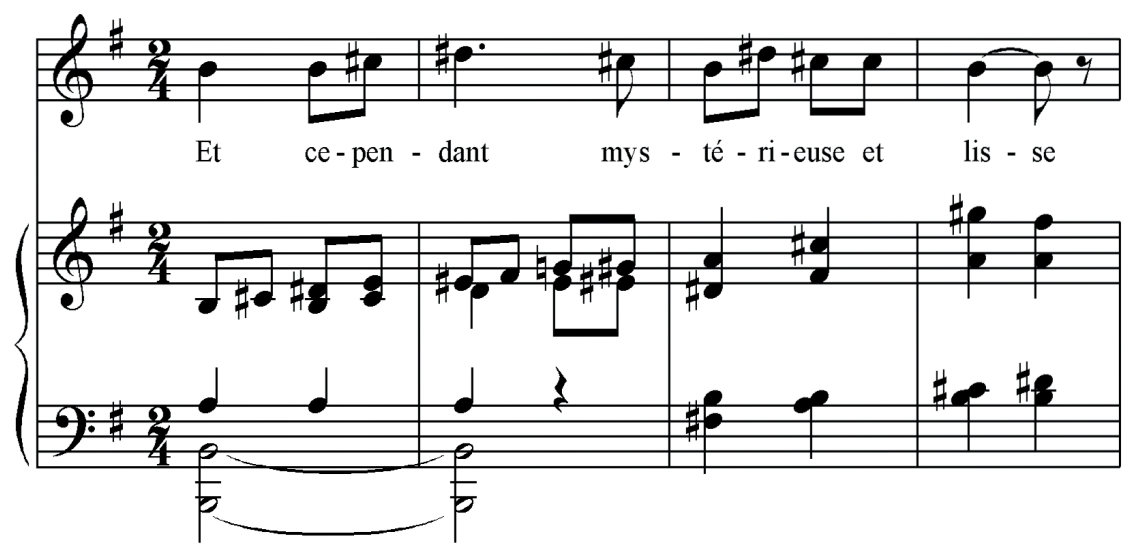

Figure 17: Claude Debussy, Pierrot, mes. 31-34.

Cette mélodie étant chantée sur une pédale de si, la référence à l'amalgame Debussy/Pierrot est toujours présente. La raison expliquant 1'appropriation de la mélodie à la voix réside dans le texte. Il est ici question de la lune, la projection astrale de Pierrot, son alter ego, mais aussi l'inspiratrice de ce poète tournant sans cesse son regard vers elle. Il va donc de soi qu'à ce moment ce soit la muse du compositeur, dont le prénom (Marie-Blanche) contient l'adjectif associé plus loin par Banville à l'astre (« la blanche lune »), qui s'empare de la mélodie - la parant d'ailleurs d'un halo de mystère et de sensualité, étant la seule présentation en augmentation rythmique du thème. La lune, en tant que miroir de Pierrot, est ici manifestement propice à jouer 
le rôle spéculaire que Michel Beaujour décèle dans maints autoportraits (Beaujour 1980, p. 20-21).

Une dernière manifestation de l'association de Debussy (si) et de Pierrot (do) se rencontre dans la coda. En effet, tandis que la chanteuse vocalise - ce chant sans texte, survenant juste après avoir mentionné "Jean-Gaspard Deburau », étant sans doute un clin d'œil à l'art sans parole des mimes - le pianiste enchaîne " Au clair de la lune » tantôt sur la tonique mi en sixtes parallèles (dont on a vu comment le si y était mis en valeur), tantôt sur do (sa tonalité d'origine) harmonisé sur le viI degré de $m i$ (mode de la). Cette alternance du si et du do, qui rappelle celle de l'introduction, devient très criante lors des trois dernières mesures, où les premières notes du thème sont jouées en imitation à la basse (rappelons qu'il s'agit de la tessiture de Debussy), sur si ou sur do. Ainsi, jusqu'à la fin de la mélodie, Debussy aura joué avec l'ambivalence propre au poème entre le personnage et l'artiste.

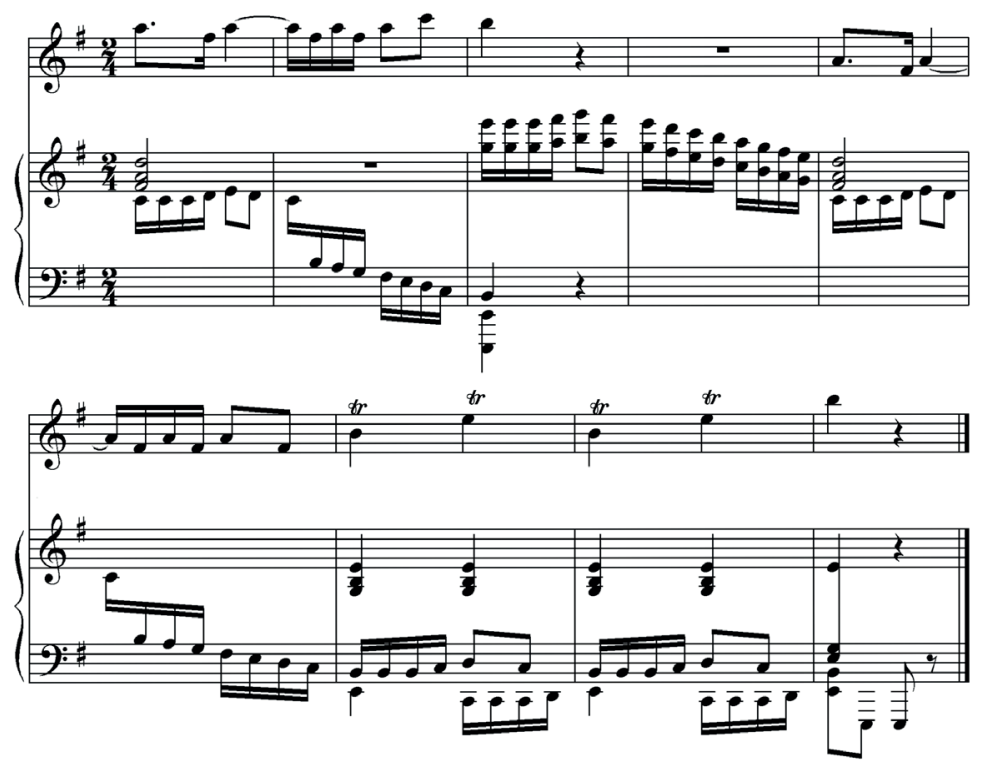

Figure 18: Claude Debussy, Pierrot, mes. 53-61.

\section{CONCLUSION}

L'analyse de Pierrot a fait apparaître que Debussy cherchait à mettre en valeur le sens du texte, et notamment celui de l'ambigüité identitaire entre Pierrot et Deburau, qu'il transpose en jouant sur la proximité de son nom avec celui du célèbre mime, par un moyen très simple : la note $s i$, qui le représente.

Il serait certainement très édifiant de passer en revue toute l'œuvre de Debussy en gardant à l'esprit le rôle que peut jouer cette note : nous y découvririons sans doute d'autres exemples de projections subjectives du compositeur dans la musique. Ainsi, par exemple, la Sonate pour violoncelle et piano en ré mineur, dont nous avons vu qu'on avait pu y détecter des éléments autobiographiques, contient-elle à la clé un si bémol - le bémol cadrant parfaitement avec l'humeur mauvaise de ce Pierrot-Debussy fâché avec la lune-musique. De même, les partitions destinées à sa femme à l'occasion de 
Noël (en 1911 et 1914), dans lesquelles Debussy se met en scène ${ }^{17}$, sont écrites en si bémol majeur.

Mais dans le cas de la mélodie Pierrot, quelles sont les conséquences de cette identification du compositeur au personnage? Parce qu'il est question du malheur amoureux du personnage lunaire assistant impuissant à l'union de Colombine et d'Arlequin, on en déduit que Debussy y expose sa souffrance de savoir sa relation amoureuse sans avenir.

Bien entendu, étant donné que cet amour est interdit car extra-conjugal, il doit rester secret. Ceci explique la manière dont le compositeur a habilement caché le message de cette œuvre : pour que la véritable nature des liens qui l'unissent à MarieBlanche Vasnier ne parvienne pas jusqu'aux oreilles de son époux, le compositeur est contraint de s'abriter derrière le sourire de façade de cette œuvre apparemment légère citant sans arrêt " Au clair de la lune".

Sans doute est-ce la raison pour laquelle Debussy n'a jamais cherché à publier cette mélodie de son vivant : il s'y jouait des enjeux trop intimes, trop personnels, pour que le compositeur veuille porter à la connaissance du monde cette confession, cette preuve d'amour désespéré, qui ne concernait que lui et sa dédicataire.

Ainsi, une fois dévoilée l'énigme de Pierrot, son habillage comique semble avoir bien pâli. Mais, après tout, n'est-ce pas en cela que réside l'Humour? Comme Nietzsche, qui affirme que "l'homme souffre si profondément qu'il a dû inventer le rire » (Nietzsche [1884-1885]1982), comme Jankélévitch, qui pense que le comique est celui « qui rit pour ne pas pleurer »(Jankélévitch [1936]2010, p. 9), nous soutenons que l'Humour repose toujours sur l'association du léger et du grave. Debussy ne nous contredirait certainement pas sur ce point, lui qui écrira à propos de sa Sonate pour flûte, alto et harpe : «je ne sais pas si on doit en rire ou en pleurer? Peut-être les deux?» (Debussy 2005, p. 2057).

\section{BIBLIOGRAPHIE}

Banville, Théodore de (1864) Les Cariatides, Paris, J. Tardieu.

Barthes, Roland (1975), Roland Barthes par Roland Barthes, Paris, Seuil.

Beaujour, Michel (1980) Miroirs d'encre, Paris, Seuil.

Bonnet, Gilles (2008), Pantomimes fin de siècle. Paris, Kimé.

Buch, Esteban (2006), Le cas Schoenberg, Paris, Gallimard.

17 Pour le Noël 1911, voir Debussy 2005, p. 1475-1477. Debussy, qui, s'il n'avait pas été musicien, aurait voulu être marin (c'est ce qu'il indique dans un questionnaire en 1888, voir p. 67), s'y représente comme un «petit mousse (irrévérencieux mais convaincu)». Pour le Noël 1914, voir ibid., p. 1858-1861). Ici, Debussy interrompt des " voix dans la rue » chantant la Marseillaise (guerre oblige) : " C'est bien évident! Mais qu'il me soit permis, en ce jour de Noël de chanter des mots plus doux que miel! Noël pour ma petite Mienne. Qu'elle veuille bien en cette année de guerre accueillir mon amour entre ses bras qui retiennent doucement, mieux que liens, plus fort que des chaînes. Qu'importe la guerre, que nous importent les clairons puisque tu es là! Que c'est ma guerre et toutes les victoires. » 
Champsaur, Félicien (1896), Pierrot et sa conscience, Paris, E. Dentu.

Debussy, Claude (1890) «Mandoline », La Revue illustrée, n 109, p. 185-188.

Debussy, Claude (1987), Monsieur Croche et autres écrits, Paris, Gallimard.

Debussy, Claude (2005), Correspondance. 1872-1918, édition établie par François Lesure et Denis Herlin, Paris, Gallimard.

Diderot, Denis ([1830]1994), Paradoxe sur le comédien, Paris, Seuil.

Fleury, Michel (1996), L’impressionnisme et la musique, Paris, Fayard.

Fulcher, Jane F. (2001), Debussy and His World, Princeton, Princeton University.

Goubault, Christian (1986), Claude Debussy, Paris, Champion.

Hennique, Léon, et Joris-Karl Huysmans (1881), Pierrot sceptique, Paris, Edouard Rouveyre.

Hirsbrunner, Theo (2002), Claude Debussy und seine Zeit, Laaber, Laaber-Verlag.

Hugounet, Paul (1889), Mimes et Pierrots, Paris, Fischbacher.

Jameux, Dominique (2002), L'école de Vienne, Paris, Fayard.

Jankélévitch, Vladimir ([1936]2010), L'ironie, Paris, Flammarion.

Jarocinski, Stefan (1971), Debussy. Impressionnisme et symbolisme, Paris, Seuil.

Koechlin, Charles (1926), "Quelques anciennes mélodies inédites de Claude Debussy », La Revue musicale, $\mathrm{n}^{\circ}$ 7, p. 115-140.

Laforgue, Jules ([1882]2008), « Pierrot sceptique », dans Pantomimes fin de siècle, textes réunis par Gilles Bonnet, Paris, Kimé, p. 71-88.

Lassauzet, Benjamin (2014), La fonction structurante du timbre dans les Préludes pour piano de Debussy, Strasbourg, Université de Strasbourg.

Lesure, François (1962), Claude Debussy, Paris, Bibliothèque Nationale.

Lockspeiser, Edward ([1962]1989), Debussy, sa vie et sa pensée, traduit de l'anglais par Léo Dilé, Paris, Fayard.

Margueritte, Paul (1882), Pierrot assassin de sa femme, Paris, P. Schmidt.

Mellers, Wilfried H. (1939), "The Final Works of Claude Debussy or Pierrot fâché avec la lune », Music and Letters, vol. 20, $\mathrm{n}^{\circ}$ 2, p. 168-176.

Mendès, Catulle (1894), La maison de la vieille, Paris, Charpentier.

Nietzsche, Friedrich ([188401885]1982), Euvres philosophiques complètes, vol. 11, Paris, Gallimard.

Palacio, Jean de (1990), Pierrot fin-de-siècle, Paris, Librairie Séguier.

Pessard, Émile (1873) Joyeusetés de bonne compagnie, Paris, Alphonse Leduc.

Pierné, Gabriel, et Paul Vidal (1926), "Souvenirs d'Achille Debussy », La Revue musicale, n 7 , p. 10-16.

Ragno, Janelle Suzanne (2005), «The Lutheran Hymn "Ein feste Burg” in Claude Debussy's Cello Sonata (1915). Motivic Variation and Structure », Thèse de doctorat, Université du Texas.

Simms, Bryan (2000), The Atonal Music of Arnold Schoenberg 1908-1923, Oxford, Oxford University Press.

Souffrin, Eileen (1960), "Debussy lecteur de Banville ", Revue de musicologie, vol. 46, n 122, p. 200-222.

Starobinski, Jean (1970), Portrait de l'artiste en saltimbanque, Genève, Albert Skira.

Valéry, Paul ([1896]1946), Monsieur Teste, Paris, Gallimard.

Vasnier, Marguerite (1926), «Debussy à 18 ans », La Revue musicale, nº 7, p. 17-22. 
Vidal, Paul (1893), Pierrot assassin de sa femme, Paris, Heugel.

Willette, Adolphe (1882), "Pierrot fumiste ", Le chat noir, n 10 (18 mars).

Willette, Adolphe (1887), Pauvre Pierrot, Paris, Léon Vanier. 